\title{
Pulmonary Stenosis in the First Year of Life
}

\author{
W. T. MUSTARD, S. C. JAIN, AND G. A. TRUSLER \\ From the Department of Surgery, Hospital for Sick Children, and the University of Toronto, Toronto, Canada
}

The purpose of this report is not only to emphasize the lethal nature of severe pulmonary stenosis in infancy but also to assess the operative results in a group of 26 infants in the first year of life. An excellent review by Luke (1966) of 36 children under 2 years of age confirms our opinion that surgical intervention should be undertaken in the first year of life. All infants were referred for operation from the Department of Cardiology under the direction of Dr. John Keith, Hospital for Sick Children, Toronto, between January 1956 and December 1965. Ten patients previously reported by Mustard, Rowe, and Firor (1960) are included as a follow-up study and those cases with pulmonary atresia and hypoplastic right ventricle are excluded.

\section{SUBJECTS}

There were 14 girls and 12 boys, their ages at operation ranging from 5 days to 1 year and their weights varying from 3.1 to $10.8 \mathrm{~kg}$. (Table I). Regardless of the age, weight and pre-operative condition of the patient, direct vision transarterial pulmonary valvotomy under mild hypothermia with inflow occlusion has been employed exclusively. All the infants presumably had a patent foramen ovale in addition to pulmonary stenosis. Right heart catheterization was performed before operation in 22 infants, and 4 were operated upon on clinical evaluation alone. Selective cine-angiocardiography was done in 17 infants. Post-operative evaluation by clinical means,

Received May 2, 1967.

TABLE I

AGE AT TIME OF OPERATION (26 PATIENTS)

\begin{tabular}{c|c}
\hline Age (mth.) & No. of patients \\
\hline & \\
\hline $1-3$ & 7 \\
$3-6$ & 4 \\
$6-9$ & 3 \\
$9-12$ & 6 \\
\hline
\end{tabular}

physical examination, radiographs, and electrocardiograms were available in 24; one has been lost to follow-up and one died. Post-operative right heart catheterization was done in 11 patients. The minimum duration of post-operative follow-up was 6 months, with a maximum of 10 years and an average of 4 years and 6 months. Seven infants were referred for surgery in the first month of life. The findings at examination are summarized in the Fig.

\section{RESULTS}

Electrocardiography. The five youngest patients, all neonates, had electrocardiographic patterns of pure left ventricular hypertrophy. The QRS axes were $+50^{\circ},+60^{\circ},+30^{\circ},+60^{\circ}$, and $+75^{\circ}$, respectively. Four other infants below 5 weeks of age showed biventricular hypertrophy, though it was predominantly right ventricular hypertrophy. In the older infants right ventricular hypertrophy was invariable. The height of $R$ in V1 exceeded 20 $\mathrm{mm}$. in 80 per cent of the infants and right axis deviation greater than $90^{\circ}$ was invariably present. In addition, a peaked $P$ wave of right atrial hypertrophy was present in 15 patients. The progression of right atrial and right ventricular hypertrophy within a few months was striking in several instances.

Haemodynamic Data. Pre-operative right heart catheterization was done in 22 patients. The right ventricular pressure varied from 115 to $186 \mathrm{~mm}$. $\mathrm{Hg}$, except in 2 infants where it was 88 and $95 \mathrm{~mm}$. $\mathrm{Hg}$, respectively. The gradient across the pulmonary valve could only be measured in 5 infants because the extremely small valve lumen did not permit passage of the catheter. Pre-operative selective cine-angiocardiography in 17 patients revealed a very stenotic valve, a right-to-left shunt through a patent foramen ovale, and a jet of contrast medium through the stenosed pulmonary valve.

\section{Surgical TechniQue}

Through a split sternal incision a transarterial approach with inflow occlusion was consistently employed. A 


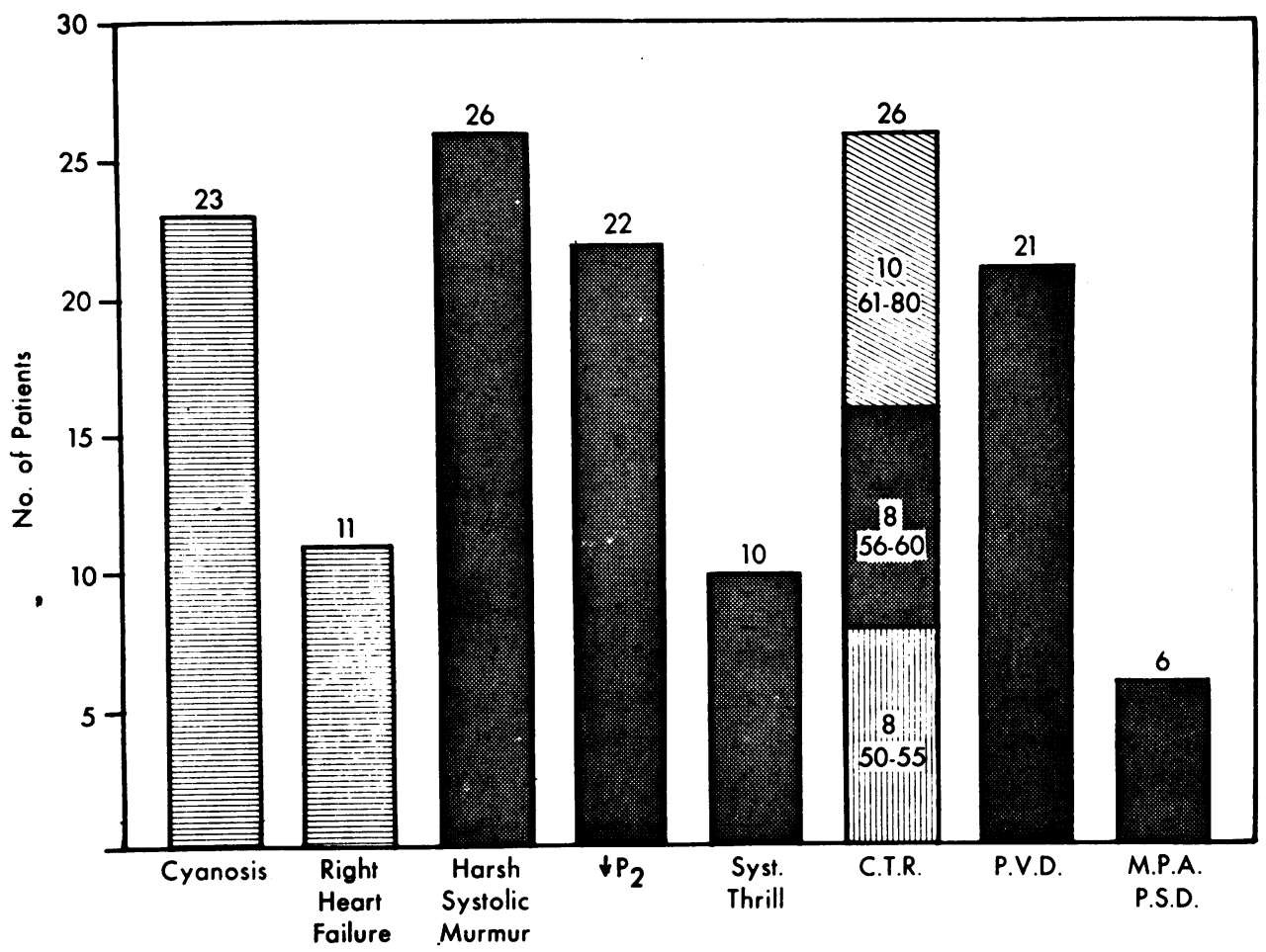

FIG.-Physical signs and radiological findings in 26 cases of pulmonary stenosis in the first year of life. $\mathbf{P}_{2}$-second pulmonary sound; C.T.R.-cardiothoracic ratio; P.V.D.-pulmonary vasculature diminished; M.P.A. P.S.D.-Post-stenotic dilatation of main pulmonary artery.

mild degree of hypothermia is now not thought to be necessary. While employing mild hypothermia, 5 developed asystole before the valve was opened and 4 went into ventricular fibrillation during inflow occlusion. All but one of these hearts resuscitated, but we felt that the cold heart was more difficult to massage, and in the past few years we have attempted to keep the infant as near normothermia as possible. The valve is consistently cone-shaped with poorly defined commissures and a small (often pin point) opening. Two bold cuts dorsal and ventral to the valve ring appear to be adequate. Occasionally the orifice is too small to permit the introduction of scissors and necessitates excision of the tip of the cone before completing the valvulotomy.

\section{RESULTS OF SURGERY}

Mild to moderate congestive heart failure occurred in 4 patients, requiring digitalis. Cyanosis persisted in 4 infants and in 2 of them re-catheterization demonstrated no gradient across the pulmonary valve but a right-to-left shunt at atrial level. The atrial septal defect was closed at 4 and 6 years of age, respectively, and the cyanosis disappeared. The other 2 children are under observation and probably fall into the group described by Oakley et al. (1964), with non-compliance of the right ventricle and reversal of shunt across a patent foramen ovale, but because they have no disability we have not operated upon them and hope the cyanosis is not significant. Right ventricular pressure studies were obtained in 11 patients and show a satisfactory reduction in pressure (Table II).

Post-operatively, immediate changes in the electrocardiogram were uncommon; a few became normal within 6 months. At the end of the follow-up period in most of the cases, the diminution of voltage in RV1 was significant and right ventricular dominance has shown great regression. At the time of the present evaluation, 7 patients had normal electrocardiograms and 17 had some degree of persistent right ventricular hypertrophy; in 15 it was mild while in 3 it was still marked. Right atrial hypertrophy, though mild and much reduced, is still present in 8 infants. A significant reduction in the degree of right axis deviation was observed, a large majority returning to normal. Pulmonary incompetence developed in 11 patients. How serious this may be is yet to be determined. 
TABLE II

PRE-OPERATIVE AND POST-OPERATIVE CATHETERIZATION DATA IN 11 PATIENTS

\begin{tabular}{c|c|c}
\hline \multirow{2}{*}{ Case No. } & \multicolumn{2}{|c}{ Right ventricular pressure (mm. $\mathbf{H g})$} \\
\cline { 2 - 3 } & Before operation & After operation \\
\hline & & \\
1 & $170 / 10$ & $35 / 10$ \\
2 & $160 / 5$ & $45 / 0$ \\
3 & $165 / 0$ & $40 / 12$ \\
4 & $135 / 15$ & $45 / 0$ \\
5 & $140 / 10$ & $50 / 0$ \\
6 & $140 / 0$ & $50 / 0$ \\
7 & $125 / 0$ & $45 / 5$ \\
8 & $125 / 15$ & $27 / 1$ \\
9 & $115 / 0$ & $50 / 0$ \\
10 & & $38 / 6$ \\
15 & & $18 / 3$ \\
\hline
\end{tabular}

\section{SUMMARY AND CONCLUSIONS}

Severe pulmonary stenosis with an intact ventricular septum in the first year of life may be an extremely dangerous lesion; its recognition and prompt surgical therapy is most rewarding.

Between January 1956 and December 1965 at the Toronto Hospital for Sick Children 26 pulmonary valvotomies were performed in infants of less than 1 year of age for pulmonary stenosis with intact ventricular septum, with one fatal outcome (4\% mortality).

Of the 26 patients, 23 had some degree of cyanosis and 11 of them had been in right heart failure. All had a systolic murmur. Five neonates had electrocardiographic evidence of pure left ventricular hypertrophy. Right atrial hypertrophy was present in 15 patients. Cardiomegaly of some degree was present in 21 infants and pulmonary vascularity was generally reduced. Post-stenotic dilatation of the pulmonary artery, so frequently present in older children, was minimal or absent. Pre-operative right heart catheterization in 22 infants revealed right ventricular pressures ranging between 115 and $186 \mathrm{~mm}$. $\mathrm{Hg}$, except in 2 cases where it was 88 and $95 \mathrm{~mm}$. Hg, respectively. Selective cine-angiocardiography was done in 17 patients and is the best method of obtaining an accurate diagnosis, because of the difficulty in catheterizing the pulmonary artery through the excessively narrow valve orifice.

Transarterial pulmonary valvotomy under direct vision with inflow occlusion and normothermia has been uniformly successful regardless of the age, size, or pre-operative condition of the patient. Early in the series mild hypothermia was employed but has now been abandoned because of the difficulty of resuscitating the cold heart. We now regard hypothermia as not only unnecessary but possibly dangerous.

\section{REFERENCES}

Luke, M. J. (1966). Valvular pulmonic stenosis in infancy. F. Pediat., 68, 90.

Mustard, W. T., Rowe, R. D., and Firor, W. B, (1960). Pulmonic stenosis in the first year of life. Results of surgery. Surgery, 47, 678 .

Oakley, C. M., Braimbridge, M. V., Bentall, H. H., and Cleland, W. P. (1964). Reversed interatrial shunt following complete relief of pulmonary valve stenosis. Brit. Heart F., 26, 662. 